Facsimile Price $\$$ Microfilm Price $\$$

Available from the Office of Technical Services Department of Commerce

Washington 25, D. C.

\title{
Wave Functions for Quadrupole Antishielding Factors*
}

Supplement to the paper: "Quadrupole Antishielding

Factors of Ions", Phys. Rev. 132, 1637 (1963).

\section{R. M. Sternheimer}

Brookhaven National Laboratory, Upton, New York

\section{INTRODUCTION}

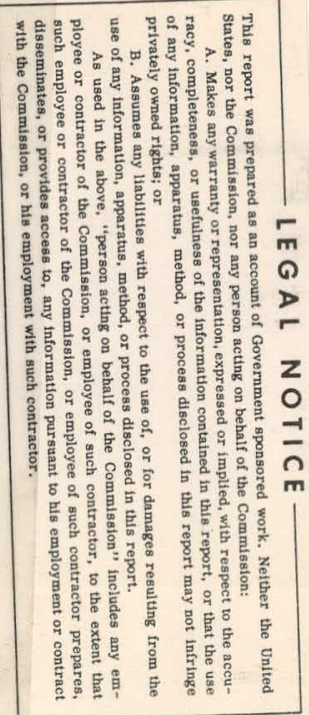

The purpose of this paper is to present tables of the

perturbed wave functions which have been recently obtained in a

2 calculation of the quadrupole antishielding factors $\gamma_{\infty}$ for the following ions: $\mathrm{F}^{-}, \mathrm{Br}^{-}, \mathrm{Rb}^{+}, \mathrm{Pr}^{3+}$, and $\mathrm{Tm}^{3+}$. The wave functions $v_{1}{ }^{\prime}(n t \rightarrow)$ which are tabulated represent the effect of the perturbation due to a nuclear quadrupole moment $Q$ on the wave functions of the outer electrons of the ion core.

The functions $v_{1} !(n b \rightarrow b)$ are the solutions of the following equation:

* Work performed under the auspices of the U.S. Atomic Energy Commission 


\section{DISCLAIMER}

This report was prepared as an account of work sponsored by an agency of the United States Government. Neither the United States Government nor any agency Thereof, nor any of their employees, makes any warranty, express or implied, or assumes any legal liability or responsibility for the accuracy, completeness, or usefulness of any information, apparatus, product, or process disclosed, or represents that its use would not infringe privately owned rights. Reference herein to any specific commercial product, process, or service by trade name, trademark, manufacturer, or otherwise does not necessarily constitute or imply its endorsement, recommendation, or favoring by the United States Government or any agency thereof. The views and opinions of authors expressed herein do not necessarily state or reflect those of the United States Government or any agency thereof. 


\section{DISCLAIMER}

Portions of this document may be illegible in electronic image products. Images are produced from the best available original document. 


$$
\left.\left[-\frac{d^{2}}{d r^{2}}+\frac{((l+1)}{r^{2}}+V_{0}-E_{0}\right] v_{1}^{\prime}(n l \rightarrow l)=u_{0}^{\prime}\left(\frac{1}{r^{3}}-\left\langle\frac{1}{r^{3}}\right)\right)_{n k^{\prime}}\right)_{1}
$$

where $u_{0}^{\prime}$ is $r$ times the unperturbed radial wave function for the ne shell; $u_{0}^{\prime}$ is normalized according to:

$$
\int_{0}^{\infty} u_{0}^{\prime 2} d r=l
$$

In Eq. (1), $\mathrm{V}_{0}$ and $\mathrm{E}_{0}$ are the unperturbed (spherical)

effective potential and the unperturbed eigenvalue for the state nl. $v_{1}^{\prime}(n l \rightarrow l)$ is completely determined by Eq. (I) and by the orthogonality condition:

$$
\int_{0}^{\infty} u_{0}^{\prime} v_{1}^{\prime}(n t \rightarrow l) d r=0
$$

We note that $v_{1}^{\prime}(n l \rightarrow l)$ is the same as the function $u_{1}^{\prime}(n l \rightarrow l)$ of reference 1 . We use the notation $v_{1}$ in order to be consistent with the notation of three previous tabulations of perturbed wave functions pertaining to the effect of the nuclear quadrupole moment. According to a method previously employed by the author, $V_{0}-E_{0}$ is obtained from the unperturbed wave function $u_{0}$ ' by means of the relation:

$$
V_{0}-E_{0}=\frac{1}{u_{0}^{T}} \frac{d^{2} u_{0}^{\prime}}{d r^{2}}-\frac{\ell(t+1)}{r^{2}}
$$


The method of solution of Eq. (1) has been described in reference 1. The unperturbed wave functions $u_{0}$ ' used in this work were the Hartree-Fock functions ${ }^{5,6}$ for $\mathrm{F}^{-}, \mathrm{Br}^{-}$, and $\mathrm{Rb}^{+}$, and the Hartree functions ${ }^{7}$ for $\mathrm{Pr}^{3+}$ and $\mathrm{Tm}^{3+}$.

The contribution to the total antishielding factor $\gamma_{\infty}$ due to the nb. $\rightarrow \ell$ mode of excitation of the ne shell is given by:

$$
\gamma_{\infty}(n t \rightarrow i)=C_{l t}^{(2)} \int_{0}^{\infty} u_{0}^{\prime} v_{1}^{\prime}(n t \rightarrow l) r^{2} d r
$$

where the coefficient $\mathrm{C}_{\ell \ell}(2)$ represents the effect of the integration over the angular variables and the summation over magnetic substates. For a completed $p$ shell, $c_{11}{ }^{(2)}=48 / 25$; for a completed a shell, $\mathrm{c}_{22}^{(2)}=16 / 7$.

The resulting functions $v_{1}^{\prime}(n p \rightarrow p)$ and $v_{1}^{\prime}(n d \rightarrow d)$ are presented in Tables I-VII. In Tables II-V pertaining to $\mathrm{Br}^{-}$and $\mathrm{Rb}^{+}$, we have also given the unperturbed functions $\mu_{0}^{\prime}(3 p), u_{0}^{\prime}(3 d)$, and $u_{0}^{\prime}(4 p)$ of Watson and Freeman, which were used in the calculations. We have also listed the values of $\left\langle r_{8}^{-3}\right\rangle_{n l}$ ( (In units $a_{H}^{-3}$ ), $\gamma_{\infty}(n l \rightarrow l)$, and $J(n l \rightarrow l)$ defined by :

$$
J(n t \rightarrow l)=\int_{0}^{\infty} u_{0}^{\prime} v_{1}^{\prime}(n t \rightarrow l) r^{-3} d r .
$$

As previously discussed in reference $8, J(\dot{n} \rightarrow l)$ enters into the calculation of the second-order quadrupole effect for the hyperfine structure. 
It is estimated that the accuracy of the functions $v_{1}$ '(nl $\left.\rightarrow l\right)$ and of the resulting values of $\gamma_{\infty}(n \ell \rightarrow \ell)$ is generally within $3 \%$. The estimated accuracy of $\mathrm{J}(\mathrm{nl} \rightarrow \ell)$ is generally within $10 \%$, with the exception of the two cases: $R b^{+} 4 p \rightarrow p$ and $\operatorname{Tm}^{3+} 5 p \rightarrow p$, where the uncertainty of the values of $v_{1}^{\prime}(n l \rightarrow l)$ near $r=0$ is somewhat greater $\left(\sim 35 \%\right.$ for $\mathrm{Rb}^{+} 4 \mathrm{p} \rightarrow \mathrm{p}_{\text {; }}$ and up to a factor of $\sim 1.8$ for $\left.\mathrm{Tm}^{3+} 5 \mathrm{p} \rightarrow \mathrm{p}\right)$. The resulting uncertainty of $J(n p \rightarrow p)$ is $\sim 30 \%$ for both cases. However, the perturbed wave functions at large $r$ are known with considerably greater precision, and as a result the values of $\gamma_{\infty}(\mathrm{np} \rightarrow \mathrm{p})$ for these two cases have been determined to $\leqslant 6 \%$. Specifically, $\left|\gamma_{\infty}(4 \mathrm{p} \rightarrow \mathrm{p})\right|$ for $\mathrm{Rb}^{+}$, which is given as 43.8 in Table I of reference 1, may be as low as 42.0, although this is considered unlikely. Similarly, for $\left|\gamma_{\infty}(5 p \rightarrow p)\right|$ of $\mathrm{Tm}^{3+}$ which is given as 67.2 , an upper limit is 72 . 
TABLE I. Wave function $v_{1}^{\prime}(2 p \rightarrow p)$ for $F^{-}$

\begin{tabular}{|c|c|c|c|}
\hline $\mathbf{r}$ & $v_{1}^{\prime}(2 p-p)$ & $r$ & $v_{1}{ }^{i}(2 p \rightarrow p)$ \\
\hline 0.01 & +0.108 & 1.4 & -0.594 \\
\hline 0.02 & 0.234 & 1.6 & -1.066 \\
\hline 0.03 & 0.369 & 1.8 & -1.407 \\
\hline 0.04 & 0.512 & 2.0 & -1.643 \\
\hline 0.05 & 0.658 & 2.2 & -1.795 \\
\hline 0.06 & 0.805 & 2.4 & -1.882 \\
\hline 0.08 & 1.101 & 2.6 & -1.919 \\
\hline 0.10 & 1.386 & 2.8 & -1.918 \\
\hline 0.12 & 1.656 & 3.0 & -1.887 \\
\hline 0.14 & 1.903 & 3.2 & -1.836 \\
\hline 0.16 & 2.131 & 3.4 & -1.771 \\
\hline 0.18 & 2.334 & 3.6 & -1.693 \\
\hline 0.20 & 2.514 & 3.8 & -1.611 \\
\hline 0.22 & 2.667 & 4.0 & -1.524 \\
\hline 0.24 & 2.803 & 4.5 & -1.303 \\
\hline 0.26 & 2.914 & 5.0 & -1.091 \\
\hline 0.28 & 3.004 & 5.5 & -0.901 \\
\hline 0.30 & 3.078 & 6.0 & -0.733 \\
\hline 0.35 & 3.187 & 6.5 & -0.592 \\
\hline 0.40 & 3.200 & 7.0 & -0.474 \\
\hline 0.45 & 3.143 & 7.5 & -0.376 \\
\hline 0.50 & 3.025 & 8.0 & -0.297 \\
\hline 0.55 & 2.864 & 8.5 & -0.234 \\
\hline 0.60 & 2.670 & 9.0 & -0.183 \\
\hline 0.7 & 2.223 & 9.5 & -0.143 \\
\hline 0.8 & 1.746 & 10.0 & -0.111 \\
\hline 0.9 & 1.274 & 11.0 & -0.068 \\
\hline 1.0 & 0.821 & 12.0 & -0.041 \\
\hline 1.1 & 0.406 & $<r^{-3}$ & 6.401 \\
\hline \multirow{2}{*}{1.2} & +0.034 & $\gamma_{\infty}$ & -23.30 \\
\hline & & $\mathrm{J}$ & +50.88 \\
\hline
\end{tabular}


TABLE II. Wave functions $v_{1}^{\prime}(3 p \rightarrow p)$ and $v_{1}^{\prime}(3 d \rightarrow \alpha)$ for $B r^{-}$. The unperturbed wave functions $u_{0}^{\prime}(3 p)$ and $u_{0}{ }^{\prime}(3 d)$ of Watson and Freeman ${ }^{6}$ are also listed.

\begin{tabular}{|c|c|c|c|c|}
\hline$r$ & $v_{1}^{\prime}(3 p \rightarrow p)$ & $v_{1}^{\prime}(3 d \rightarrow d)$ & $u_{0}^{\prime}(3 p)$ & $u_{0}^{\prime}(3 d)$ \\
\hline 0.01 & +2.77 & +0.0187 & +0.0398 & +0.0006 \\
\hline 0.02 & 5.36 & +0.0773 & 0.1338 & 0.0043 \\
\hline 0.03 & 7.21 & 0.171 & 0.2526 & 0.0132 \\
\hline 0.04 & 8.26 & 0.292 & 0.3766 & 0.0281 \\
\hline 0.05 & 8.55 & 0.436 & 0.4927 & 0.0494 \\
\hline 0.06 & 8.17 & 0.597 & 0.5930 & 0.0770 \\
\hline 0.07 & 7.25 & .0 .767 & 0.6727 & 0.1105 \\
\hline 0.08 & 5.90 & 0.943 & 0.7299 & 0.1492 \\
\hline 0.09 & 4.24 & 1.116 & 0.7641 & 0.1924 \\
\hline 0.10 & +2.36 & 1.287 & 0.7761 & 0.2394 \\
\hline 0.12 & -1.71 & 1.608 & 0.7397 & 0.3415 \\
\hline 0.14 & -5.76 & $1.882^{\circ}$ & 0.6368 & 0.4500 \\
\hline 0.16 & -9.41 & 2.102 & 0.4854 & 0.5598 \\
\hline 0.18 & -12.46 & 2.263 & 0.302 .3 & 0.6671 \\
\hline 0.20 & -14.80 & 2.365 & +0.1020 & 0.7691 \\
\hline 0.22 & -16.42 & 2.413 & -0.1036 & 0.8636 \\
\hline 0.24 & -17.39 & 2.412 & -0.3056 & 0.9493 \\
\hline 0.26 & -17.79 & 2.369 & -0.4972 & 1.0256 \\
\hline 0.28 & -17.68 & 2.289 & -0.6740 & 1.0919 \\
\hline 0.30 & -17.14 & 2.178 & -0.8329 & 1.1484. \\
\hline 0.35 & -14.53 & 1.803 & -1.1449 & 1.2484 \\
\hline 0.40 & -10.86 & 1.353 & -1.3378 & 1.2967 \\
\hline 0.45 & -6.93 & 0.883 & -1.4296 & 1.3031 \\
\hline 0.50 & -3.22 & 0.433 & -1.4437 & 1.2787 \\
\hline 0.55 & -0.01 & +0.022 & -1.4027 & 1.2317 \\
\hline 0.60 & +2.57 & -0.336 & -1.3255 & 1.1699 \\
\hline 0.7 & 6.34 & -0.903 & -1.1117 & 1.0233 \\
\hline 0.8 & 8.10 & -1.258 & -0.8934 & 0.8698 \\
\hline
\end{tabular}


TABLE II (cont'd).

\begin{tabular}{|c|c|c|c|c|}
\hline$r$ & $v_{1}^{\prime}(3 p-p)$ & $v_{1}{ }^{\prime}(3 d \rightarrow d)$ & $u_{0}^{\prime}(3 p)$ & $u_{0}^{\prime}(3 d)$ \\
\hline 0.9 & 8.40 & -1.443 & -0.6899 & 0.7252 \\
\hline 1.0 & 7.86 & -1.501 & -0.5195 & 0.5964 \\
\hline 1.1 & 6.92 & -1.473 & -0.3840 & 0.4855 \\
\hline 1.2 & 5.85 & -1.389 & -0.2799 & 0.3921 \\
\hline 1.4 & 3.77 & -1.136 & -0.1450 & 0.2517 \\
\hline 1.6 & 2.31 & -0.873 & -0.0743 & 0.1595 \\
\hline 1.8 & 1.40 & -0.649 & -0.0385 & 0.1007 \\
\hline 2.0 & 0.855 & -0.472 & -0.0205 & 0.0636 \\
\hline 2.2 & 0.526 & -0.340 & -0.0112 & 0.0404 \\
\hline 2.4 & 0.317 & -0.243 & -0.0062 & 0.0258 \\
\hline 2.6 & 0.190 & -0.173 & -0.0033 & 0.0166 \\
\hline 2.8 & & -0.123 & & 0.0107 \\
\hline 3.0 & & -0.086 & & 0.0069 \\
\hline 3.2 & & -0.060 & & 0.0045 \\
\hline 3.4 & & -0.042 & & 0.0029 \\
\hline 3.6 & & -0.029 & & 0.0019 \\
\hline$\left\langle r^{-3}\right\rangle_{\text {ns }}$ & 181.3 & 23.08 & $\cdots$ & -.... \\
\hline$\gamma_{\infty}$ & -4.51 & -1.627 & $\cdots$ & $\cdots$ \\
\hline $\mathrm{J}$ & +4545 & +66.50 & $\ldots$ & -..... \\
\hline
\end{tabular}


TABLE III. Wave function $v_{1}{ }^{\prime}(4 \mathrm{p}-\mathrm{p})$ for $\mathrm{Br}^{-}$.

\begin{tabular}{lll}
\hline $\mathbf{r}$ & $v_{1}^{\prime}(4 p-p)$ & $u_{0}^{\prime}(4 p)$ \\
\hline 0.01 & +0.68 & +0.00940 \\
0.02 & 1.35 & 0.03190 \\
0.03 & 1.86 & 0.06023 \\
0.04 & 2.17 & 0.08973 \\
0.05 & 2.30 & 0.1173 \\
0.06 & 2.26 & 0.1409 \\
0.07 & 2.08 & 0.1594 \\
0.08 & 1.77 & 0.1725 \\
0.09 & 1.37 & 0.1798 \\
0.10 & +0.91 & 0.1817 \\
0.12 & -0.13 & 0.1707 \\
0.14 & -1.20 & 0.1435 \\
0.16 & -2.19 & 0.1047 \\
0.18 & -3.04 & 0.0585 \\
0.20 & -3.71 & +0.0086 \\
0.22 & -4.18 & -0.0418 \\
0.24 & -4.46 & -0.0905 \\
0.26 & -4.57 & -0.1357 \\
0.28 & -4.52 & -0.1763 \\
0.30 & -4.33 & -0.2114 \\
0.35 & -3.38 & -0.2733 \\
0.40 & -1.98 & -0.2991 \\
0.45 & -0.36 & -0.2936 \\
0.50 & +1.28 & -0.2635 \\
0.55 & 2.84 & -0.2154 \\
0.60 & 4.24 & -0.1549 \\
0.7 & 6.56 & -0.0152 \\
0.8 & 8.10 & +0.1292 \\
0.9 & 9.00 & 0.2637 \\
& & \\
0.9 & \\
0.04
\end{tabular}


TABLE III(cont'd).

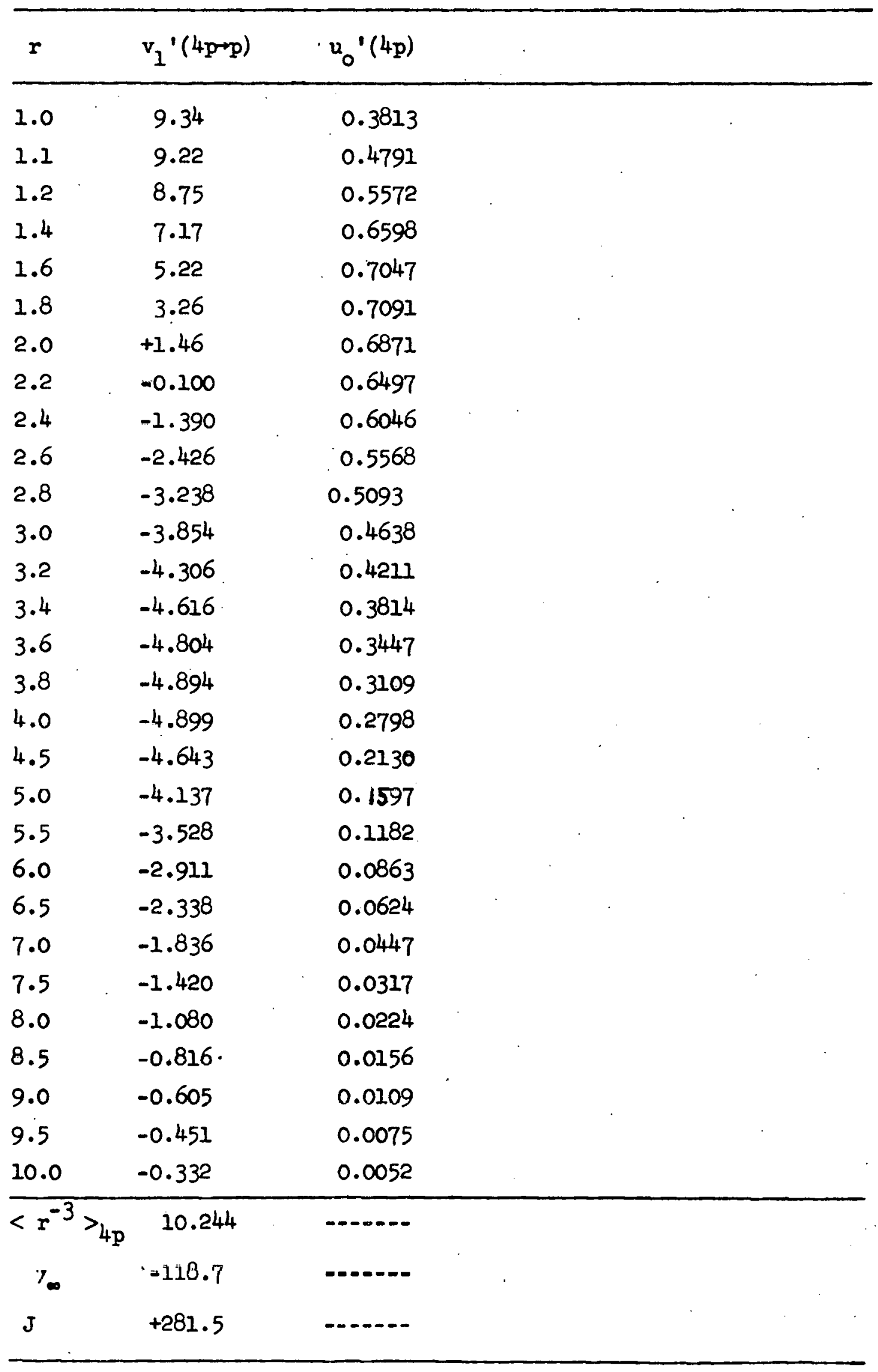


TABLE IV. Wave functions $v_{1}^{\prime}(3 p-p)$ and $v_{1}{ }^{\prime}(3 d \rightarrow d)$ for $\mathrm{Rb}^{+}$.

The unperturbed wave functions $u_{0}^{\prime}(3 p)$ and $u_{0}{ }^{\prime}(3 d)$ of Watson and Freeman ${ }^{6}$ are also listed.

\begin{tabular}{|c|c|c|c|c|}
\hline$r$ & $v_{1}{ }^{\prime}(3 p \rightarrow p)$ & $v_{1}{ }^{\prime}(3 d-d)$ & $u_{0}^{\prime}(3 p)$ & $u_{0}^{\prime}(3 d)$ \\
\hline 0.01 & +3.28 & +0.0247 & +0.0470 & +0.0008 \\
\hline 0.02 & 6.27 & 0.1015 & 0.1561 & 0.0057 \\
\hline 0.03 & 8.29 & 0.2218 & 0.2918 & 0.0171 \\
\hline 0.04 & 9.33 & 0.3746 & 0.4302 & 0.0361 \\
\hline 0.05 & 9.43 & 0.5552 & 0.5565 & 0.0632 \\
\hline 0.06 & 8.74 & 0.7534 & 0.6616 & 0.0980 \\
\hline 0.07 & 7.41 & 0.9596 & 0.7410 & 0.1397 \\
\hline 0.08 & 5.62 & 1.1683 & 0.7929 & 0.1876 \\
\hline 0.09 & 3.49 & 1.3728 & 0.8174 & 0.2406 \\
\hline 0.10 & +1.17 & 1.568 & 0.8161 & 0.2976 \\
\hline 0.12 & -3.80 & 1.923 & 0.7456 & 0.4199 \\
\hline 0.14 & -8.50 & 2.207 & 0.6033 & 0.5472 \\
\hline 0.16 & -12.56 & 2.416 & 0.4121 & 0.6735 \\
\hline 0.18 & -15.74 & 2.547 & +0.1923 & 0.7942 \\
\hline 0.20 & -18.00 & 2.604 & -0.0393 & 0.9062 \\
\hline 0.22 & -19.37 & 2.597 & -0.2695 & 1.0073 \\
\hline 0.24 & -19.99 & 2.534 & -0.4890 & 1.0964 \\
\hline 0.26 & -19.92 & 2.422 & $=0.6911$ & 1.1728 \\
\hline 0.28 & -19.29 & 2.271 & -0.8718 & 1.2365 \\
\hline 0.30 & -18.20 & 2.091 & -1.0289 & 1.2880 \\
\hline 0.35 & -14.11 & 1.555 & -1.3169 & 1.3677 \\
\hline 0.40 & -9.23 & 0.976 & -1.4686 & 1.3881 \\
\hline 0.45 & -4.46 & +0.418 & -1.5127 & 1. .3639 \\
\hline 0.50 & -0.28 & -0.082 & -1.4798 & 1.3084 \\
\hline 0.55 & +3.09 & -0.508 & -1.3968 & 1.2325 \\
\hline 0.60 & 5.603 & -0.852 & -1.2843 & 1.1448 \\
\hline 0.7 & 8.46 & -1.335 & -1.0272 & 0.9573 \\
\hline 0.8 & 9.22 & -1.563 & -0.7807 & $\cdot 0.7773$ \\
\hline 0.9 & 8.70 & -1.612 & -0.5731 & 0.6185 \\
\hline
\end{tabular}


TABLE IV (cont'd).

\begin{tabular}{|c|c|c|c|c|}
\hline $\mathbf{r}$ & $v_{1}^{\prime}(3 p \rightarrow p)$ & $v_{1}^{\prime}(3 d-d)$ & $u_{0}^{\prime}(3 p)$ & $u_{0}^{\prime} !(3 d)$ \\
\hline 1.0 & 7.54 & -1.545 & -0.4104 & 0.4850 \\
\hline 1.1 & 6.19 & -1.413 & -0.2887 & 0.3763 \\
\hline 1.2 & 4.92 & -1.253 & -0.2007 & 0.2897 \\
\hline 1.4 & 2.97 & -0.912 & -0.0954 & 0.1691 \\
\hline 1.6 & 1.67 & -0.631 & -0.0454 & 0.0977 \\
\hline 1.8 & 0.934 & -0.424 & -0.0220 & 0.0565 \\
\hline 2.0 & 0.515 & -0.281 & -0.0108 & 0.0328 \\
\hline 2.2 & 0.279 & -0.184 & -0.0052 & 0.0191 \\
\hline 2.4 & 0.151 & -0.119 & -0.0023 & 0.0112 \\
\hline 2.6 & & -0.076 & & 0.0066 \\
\hline 2.8 & & -0.049 & & 0.0039 \\
\hline 3.0 & & -0.031 & & 0.0023 \\
\hline 3.2 & : & -0.020 & & 0.0013 \\
\hline$\left\langle r^{-3}\right\rangle_{n s}$ & 228.1 & 30.61 & --.-- & -..- \\
\hline$\gamma_{\infty}$ & -3.93 & -1.231 & -..- & $\cdots$ \\
\hline $\mathrm{J}$ & +6006 & +89.70 & $\cdots$ & -... \\
\hline
\end{tabular}


TABLE V. Wave function $v_{1}^{\prime}(4 p \rightarrow p)$ for $\mathrm{Rb}^{+}$.

\begin{tabular}{|c|c|c|c|c|c|}
\hline$r$ & $v_{1}{ }^{\prime}(4 p \rightarrow p)$ & $u_{0}^{\prime}(4 p)$ & $r$ & $v_{1}:(4 p-p)$ & $u_{0}^{\prime}(4 p)$ \\
\hline 0.01 & $\infty 0.97$ & +0.02404 & \pm .2 & 6.84 & 0.7895 \\
\hline 0.02 & 1.83 & 0.04668 & 1.4 & 3.57 & 0.8459 \\
\hline 0.03 & 2.40 & 0.08719 & 1.6 & +0.67 & 0.8285 \\
\hline 0.04 & 2.66 & 0.12843 & 1.8 & -1.61 & 0.7684 \\
\hline 0.05 & 2.65 & 0.1659 & 2.0 & -3.23 & 0.6878 \\
\hline 0.06 & 2.40 & 0.1967 & 2.2 & -4.26 & 0.6008 \\
\hline 0.07 & 1.96 & 0.2196 & 2.4 & -4.83 & 0.5157 \\
\hline 0.08 & 1.38 & 0.2340 & 2.6 & -5.04 & 0.4370 \\
\hline 0.09 & +0.70 & 0.2400 & 2.8 & -4.99 & 0.3665 \\
\hline 0.10 & -0.03 & 0.2379 & 3.0 & $-4 \cdot 76$ & 0.3047 \\
\hline 0.12 & -1.54 & 0.2130 & 3.2 & -4.42 & 0.2515 \\
\hline 0.14 & -2.93 & 0.1662 & 3.4 & -4.02 & 0.2061 \\
\hline 0.16 & -4.10 & 0.1049 & 3.6 & -3.60 & 0.1679 \\
\hline 0.18 & -4.96 & +0.0356 & 3.8 & -3.17 & 0.1360 \\
\hline 0.20 & -5.50 & -0.0361 & 4.0 & -2.76 & 0.1095 \\
\hline 0.22 & -5.73 & -0.1062 & 4.5 & -1.83 & 0.06235 \\
\hline 0.24 & $-5 \cdot 70$ & -0.1713 & 5.0 & -1.15 & 0.03459 \\
\hline 0.26 & -5.43 & -0.2293 & 5.5 & -0.700 & 0.01882 \\
\hline 0.28 & -4.95 & -0.2790 & 6.0 & -0.415 & 0.01011 \\
\hline 0.30 & $-4 \cdot 31$ & -0.3195 & 6.5 & -0.241 & 0.00540 \\
\hline 0.35 & -2.20 & -0.3801 & 7.0 & $=0.138$ & 0.00288 \\
\hline 0.40 & +0.22 & -0.3874 & 7.5 & -0.078 & 0.00154 \\
\hline 0.45 & 2.61 & -0.3519 & 8.0 & -0.047 & 0.00083 \\
\hline 0.50 & 4.76 & -0.2849 & 8.5 & -0.027 & 0.00045 \\
\hline 0.55 & 6.55 & -0.1970 & 9.0 & -0.016 & 0.00024 \\
\hline 0.60 & 7.93 & -0.0968 & & & \\
\hline 0.7 & 9.78 & +0.1150 & \multirow{3}{*}{$\begin{array}{c}<r^{-} \\
\gamma_{\infty}\end{array}$} & 20.21 & $--\infty--$ \\
\hline 0.8 & 10.49 & 0.3151 & & -42.0 & \\
\hline 0.9 & 10.34 & 0.4861 & & & \\
\hline 1.0 & 9.54 & 0.6217 & $\mathbf{J}$ & +516 & $\ldots$ \\
\hline 1.1 & 8.30 & 0.7218 & & & \\
\hline
\end{tabular}


TABLE vI. Wave functions $v_{1}{ }^{\prime}(4 p \rightarrow p)$ and $v_{1}{ }^{\prime}(5 p \rightarrow p)$ for $\mathrm{Pr}^{3+}$.

\begin{tabular}{|c|c|c|c|c|c|}
\hline$r$ & $v_{1}{ }^{\prime}(4 p \rightarrow p)$ & $v_{1}^{\prime}(5 p-p)$ & $r$ & $v_{1}^{\prime}(4 p \rightarrow p)$ & $\nabla_{1}^{\prime}(5 p \rightarrow p)$ \\
\hline 0.01 & +6.15 & +1.86 & 1.2 & -11.65 & -16.65 \\
\hline 0.02 & 10.32 & 2.80 & 1.4 & -7.96 & -11.15 \\
\hline 0.03 & 10.30 & 2.62 & 1.6 & -4.89 & -4.88 \\
\hline 0.04 & 7.78 & 1.61 & 1.8 & -2.81 & +0.42 \\
\hline 0.05 & +3.63 & +0.11 & 2.0 & -1.542 & 4.39 \\
\hline 0.06 & -1.30 & -1.56 & 2.2 & -0.823 & 7.03 \\
\hline 0.07 & -6.31 & -3.25 & 2.4 & -0.431 & 8.49 \\
\hline 0.08 & -10.92 & -4.60 & 2.6 & -0.223 & 9.09 \\
\hline 0.09 & -14.81 & -5.63 & 2.8 & -0.114 & 9.03 \\
\hline 0.10 & -17.81 & -6.31 & 3.0 & -0.058 & 8.58 \\
\hline 0.12 & -20.85 & -6.58 & 3.2 & -0.030 & 7.83 \\
\hline 0.14 & -20.42 & -5.69 & 3.4 & -0.015 & 7.00 \\
\hline 0.16 & -17.23 & -4.17 & 3.6 & & 6.12 \\
\hline 0.18 & -12.11 & -2.20 & 3.8 & & 5.27 \\
\hline 0.20 & -5.83 & +0.03 & 4.0 & & 4.49 \\
\hline 0.22 & +0.92 & 2.29 & 4.5 & & 2.743 \\
\hline 0.24 & 7.52 & 4.47 & 5.0 & . & $i .585$ \\
\hline 0.26 & 13.61 & 6.34 & 5.5 & & 0.880 \\
\hline 0.28 & 18.91 & 7.82 & 6.0 & & 0.471 \\
\hline 0.30 & 23.29 & 8.90 & 6.5 & & 0.244 \\
\hline 0.35 & 30.12 & 10.89 & 7.0 & & 0.122 \\
\hline 0.40 & $31 \cdot 31$ & 10.70 & 7.5 & & 0.061 \\
\hline 0.45 & 28.39 & 8.60 & 8.0 & 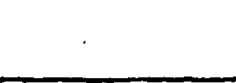 & 0.031 \\
\hline 0.50 & 22.95 & 5.18 & $\left\langle\mathrm{r}^{-3}\right\rangle_{n \ell}$ & 285.6 & 40.10 \\
\hline 0.55 & 16.36 & +1.26 & & -8.81 & \\
\hline 0.60 & +9.66 & -2.71 & $\gamma_{\infty}$ & -8.01 & -69.7 \\
\hline 0.7 & -1.73 & -10.27 & & & \\
\hline 0.8 & -9.12 & -15.65 & & & \\
\hline 0.9 & -12.82 & -18.58 & & & \\
\hline 1.0 & -13.84 & -19.38 & & & \\
\hline 1.1 & -13.18 & -18.59 & & & \\
\hline
\end{tabular}


TABLE VII. Wave functions $v_{1}{ }^{\prime}(4 p \rightarrow p)$ and $v_{1}{ }^{\prime}(5 p \rightarrow p)$ for $\mathrm{m}^{3+}$.

\begin{tabular}{|c|c|c|c|c|c|}
\hline$r$ & $v_{1}^{\prime}(4 p-p)$ & $v_{1}^{\prime}(5 p \rightarrow p)$ & $\dot{r}$ & $v_{1}^{\prime}(4 p-p)$ & $v_{1}^{\prime}(5 p \rightarrow p)$ \\
\hline 0.01 & +8.53 & +4.36 & 1.4 & -4.65 & -3.20 \\
\hline 0.02 & 15.16 & 6.30 & 1.6 & -2.278 & +3.63 \\
\hline 0.03 & 13.76 & 6.14 & 1.8 & -1.065 & 8.18 \\
\hline 0.04 & 8.30 & 4.36 & 2.0 & -0.482 & 10.74 \\
\hline 0.05 & +0.66 & +1.61 & 2.2 & -0.215 & 11.78 \\
\hline 0.06 & -7.55 & -1.53 & 2.4 & -0.095 & 11.78 \\
\hline 0.07 & -15.05 & -4.52 & 2.6 & -0.042 & 11.09 \\
\hline 0.08 & -21.11 & -7.12 & 2.8 & -0.018 & 10.01 \\
\hline 0.09 & -25.31 & -9.10 & 3.0 & & 8.76 \\
\hline 0.10 & -27.48 & -10.34 & 3.2 & & 7.49 \\
\hline 0.12 & -26.32 & -10.65 & 3.4 & & 6.27 \\
\hline 0.14 & -19.76 & -8.67 & 3.6 & & 5.16 \\
\hline 0.16 & -9.97 & -5.06 & 3.8 & & 4.19 \\
\hline 0.18 & +1.06 & -0.73 & 4.0 & & 3.36 \\
\hline 0.20 & 11.86 & +3.71 & 4.5 & & 1.85 \\
\hline 0.22 & 21.39 & 7.77 & 5.0 & & 0.97 \\
\hline 0.24 & 29.10 & 21.14 & 5.5 & & 0.493 \\
\hline 0.26 & 34.72 & 13.67 & 6.0 & & 0.250 \\
\hline 0.28 & 38.29 & 15.35 & 6.5 & & 0.125 \\
\hline 0.30 & 40.05 & 16.20 & 7.0 & & 0.063 \\
\hline 0.35 & 38.51 & 15.33 & & & \\
\hline 0.40 & 30.74 & 10.98 & $\left\langle r^{-3}\right\rangle_{n e}$ & 518.2 & 61.87 \\
\hline 0.45 & 20.33 & +4.67 & & & \\
\hline 0.50 & 9.83 & -2.24 & $\gamma_{\infty}$ & -6.79 & -67.2 \\
\hline 0.5 .5 & +0.69 & -8.85 & & & \\
\hline 0.60 & -6.52 & -14.62 & & & \\
\hline 0.7 & -15.33 & -22.47 & & & \\
\hline 0.8 & -17.99 & -25.31 & & & . \\
\hline 0.9 & -17.05 & -24.27 & & & \\
\hline 1.0 & -14.56 & -21.04 & & & \\
\hline 1.1 & -11.61 & -16.72 & & & \\
\hline $1 . \hat{c}$ & -8.84 & -11.99 & & & \\
\hline
\end{tabular}


REFERENCES

1. R.M. Sternheimer, Phys. Rev. 132,

(1963)

2. R.M. Stermhelmer, Phys. Rev. 84,244 (1951); H.M. Foley,

R.M. Stermheimer, and D. Tycko, Phys. Rev. 93, 734 (1954);

R.M. Sternheimer and H.M. Foley, Phys. Rev. 102, 731 (1956);

R.M. Sternhelmer, Phys. Rev. 130, 1423 (1963).

3. R.M. Stermheimer, Documents No. 6044,7341 , and 7475 , ADI Auxiliary Publication Project, Library of Congress, Washington, D.C.

4. R.M. Sternheimer, Phys. Rev. 96, 951 (1954); 107, 1565 (1957); $115 ; 1198$ (1959).

5. C. Froese, Proc. Cambridge Phil. Soc. 53, 206 (1957).

6. R.E. Watson and A.J. Freeman, Phys. Rev. 124, 1117 (1961).

7. Ẹc. Ridley, Proc. Cambriage Phil. Soc. 56, 41 (1960).

8. R.M. Sternheimer, Phys. Rev. 127, 812 (1962). 